\section{Thrombektomie nach 6 - 24 Stunden: Viele Patienten profitieren}

Nogueira RG et al. Thrombectomy 6 to 24 Hours after Stroke with a Mismatch between Deficit and Infarct. N Engl ] Med 2018; 378: 11-21

Bei einem ischämischen Schlaganfall sollte eine Thrombektomie so früh wie möglich erfolgen. Studien belegen einen Nutzen für die ersten 6 Stunden nach Symptombeginn. Was aber, wenn mehr als 6 Stunden vergangen sind oder nicht bekannt ist, wann die ersten Symptome auftraten? Die DAWN-Studie hat untersucht, ob Patienten mit einer starken Diskrepanz zwischen klinischen Symptomen und Infarktgröße auch von einer späteren Intervention profitieren.

Wann ging es dem Patienten zuletzt gut? Dieser Zeitpunkt wird in der Regel für die Festlegung verwendet, wie lange der Schlaganfall zurückliegt. Wenn der Patient mit Schlaganfallsymptomen aus dem Schlaf erwacht oder erkrankt aufgefunden wird, überschätzt diese Methode aber möglicherweise die Dauer der Symptome. Auch Patienten mit ischämischem Hirngewebe, das noch nicht abgestorben ist, könnten profitieren, wenn die 6-StundenRegel nicht streng eingehalten wird. Letzterer Fall liegt dann vor, wenn die klinischen Symptome einen deutlich größeren Schaden vermuten lassen, als er im Hirnscan sichtbar ist. Auf diese Patienten konzentriert sich die prospektive, randomisierte Multizenter-Studie DAWN (DWI or CTP Assessment with Clinical Mismatch in the Triage of Wake-Up and Late Presenting Strokes Undergoing Neurointervention with Trevo).

206 Patienten mit Verschluss der intrakranialen A. carotis interna oder der proximalen $A$. cerebri media, denen es zuletzt 6-24 Stunden vor Aufnahme in die Studie gut ging, bekamen randomisiert entweder eine Thrombektomie plus die Standardversorgung oder nur die Standardversorgung (Kontrollgruppe). Endpunkte waren der Grad der Behinderung auf der nutzengewichteten modifizierten Rankin-Skala (von $0=$ Tod bis $10=$ keine Symptome oder Behinderung) und die funktionelle
Unabhängigkeit (Grad 0, 1 oder 2 auf der modifizierten Rankin-Skala von 0 bis 6 , wobei höhere Werte eine größere Einschränkung bedeuten).

\section{Jede(r) Zweite erreicht funktionelle Unabhängigkeit}

Nach 90 Tagen war der mittlere Wert auf der nutzengewichteten modifizierten Rankin-Skala 5,5 in der ThrombektomieGruppe und 3,4 in der Kontrollgruppe. Funktionelle Unabhängigkeit erreichten 49\% der Patienten in der ThrombektomieGruppe, aber nur $13 \%$ in der Kontrollgruppe. Symptomatische Hirnblutungen waren in beiden Gruppen ähnlich häufig (6\% vs. $3 \%, P=0,50)$, auch die 90 -TageMortalität unterschied sich nicht wesentlich (19\% vs. $18 \%, P=1,00$ ).

Diese Ergebnisse sind eindrucksvoll. Eine Zwischenanalyse der Studie führte dazu, dass die Rekrutierung neuer Patienten nach 31 Monaten gestoppt wurde. Für 2 Patienten, die einer Thrombektomie unterzogen wurden, hatte 1 Patient mehr nach 90 Tagen einen besseren Wert bei der Beurteilung der Einschränkungen. Für 2,8 thrombektomierte Patienten hatte einer mehr nach 90 Tagen funktionelle Unabhängigkeit, konnte sich also im Alltag selbst versorgen. Der Anteil der Patienten mit funktioneller Unabhängigkeit in der Thrombektomie-Gruppe war ähnlich hoch wie in Studien mit früher Thrombektomie.

\section{FAZIT}

Nach den Ergebnissen dieser Studie könnten viele Schlaganfallpatienten auch nach dem 6-Stunden-Zeitfenster von einer Thrombektomie profitieren. In retrospektiven Studien erfüllte etwa ein Drittel aller Patienten mit Verschluss eines vorderen Hirngefäßes, die sich zwischen 6 und 24 Stunden nach Symptombeginn vorstellen, die Einschlusskriterien, die für die DAWN-Studie verwendet wurden.

Dr. Nina Drexelius, Hamburg 